\title{
Distribuição e morfologia do sistema radicular de Eucalyptus dunnii em resposta à aplicação de fósforo
}

\author{
Distribution and morphology of root system of Eucalyptus dunnii in response to phosphorus fertilization
}

\section{Luciana Patricia Rosa Dias ${ }^{1 *}$, Luciano Colpo Gatiboni ${ }^{1}$, Gustavo Brunetto ${ }^{2}$, Bruna Arruda ${ }^{3}$ e Muriá Mussi Costa ${ }^{1}$}

Recebido em 12/09/2016 / Aceito em 18/01/2017

\section{RESUMO}

A utilização de fosfatos solúveis e fosfatos naturais é prática usual na adubação de eucalipto. Como a adubação fosfatada é localizada próximo às plantas, o fósforo $(\mathrm{P})$ pode interferir no crescimento e na morfologia do sistema radicular. O objetivo do trabalho foi avaliar o crescimento e as características morfológicas do sistema radicular de Eucalyptus dunnii com o uso de fosfato solúvel e natural. As amostras foram coletadas no ano de 2013 de um experimento instalado em 2010 no município de Otacílio Costa, SC. Foram coletadas amostras de quatro tratamentos: 1) testemunha sem $\mathrm{P}$; 2) adubação com $60 \mathrm{~kg} \mathrm{ha}^{-1} \mathrm{de}_{2} \mathrm{O}_{5}$, na forma de fosfato natural; 3) $75 \mathrm{~kg} \mathrm{ha}^{-1}$ de $\mathrm{P}_{2} \mathrm{O}_{5}$, na forma de superfosfato triplo e; 4) $60 \mathrm{~kg} \mathrm{ha}^{-1}$ de $\mathrm{P}_{2} \mathrm{O}_{5}$ de fosfato natural mais $75 \mathrm{~kg} \mathrm{ha}^{-1} \mathrm{de}$ $\mathrm{P}_{2} \mathrm{O}_{5}$ de fosfato solúvel, sendo essas doses escolhidas por representar a prática usual nos reflorestamentos da região. A coleta de raízes ocorreu 28 meses após o plantio das mudas e aplicação dos tratamentos com o auxílio de uma sonda de $40 \mathrm{~mm}$ de diâmetro. As amostras de solo e raízes foram coletas em três distâncias a partir do fuste $(10,60$ e $120 \mathrm{~cm})$ e em duas profundidades $(0-10$ e $10-20 \mathrm{~cm})$. As raízes foram separadas em duas classes de diâmetro: 0 a $2 \mathrm{~mm}$ e 2 a $5 \mathrm{~mm}$. Foram caracterizadas a massa seca de raízes, raio radicular, comprimento, volume e área radicular. No solo, foi determinado o teor de $\mathrm{P}$ disponível. O fosfato natural não estimulou o crescimento radicular e o fosfato solúvel foi a fonte que proporcionou melhor crescimento e desenvolvimento de raízes finas no Eucalyptus dunnii, sendo que a maior concentração dessas raízes está localizada próximo ao fuste e na camada mais superficial do solo.
PALAVRAS-CHAVE: raízes de absorção, eucalipto, adubação fosfatada.

\section{ABSTRACT}

The use of soluble phosphates and rock phosphates is a normal practice in Eucalyptus fertilization. As the phosphorus fertilizer is located near the plant, phosphorus (P) may interfere with the growth and morphology of the root system. The objective of this work was to evaluate the growth and morphological characteristics of root system of Eucalyptus dunnii using soluble and rock phosphate. Samples were collected in 2013 from an experiment established in 2010 in the city of Otacílio Costa, SC. Samples were collected from four treatments: 1) control without $\mathrm{P}$; 2) fertilization with $60 \mathrm{~kg} \mathrm{ha}^{-1}$ of $\mathrm{P}_{2} \mathrm{O}_{5}$ in the form of phosphate rock; 3) $75 \mathrm{~kg} \mathrm{ha}^{-1}$ of $\mathrm{P}_{2} \mathrm{O}_{5}$ in the form of triple superphosphate and; 4) $60 \mathrm{~kg} \mathrm{ha}^{-1}$ rock phosphate $\mathrm{P}_{2} \mathrm{O}_{5}$ plus $75 \mathrm{~kg} \mathrm{ha}^{-1} \mathrm{P}_{2} \mathrm{O}_{5}$ soluble phosphate (these doses being chosen because it represents the usual practice in the reforestation of the region). The collection of roots occurred 28 months after planting of the seedlings and application of treatment with a 40 $\mathrm{mm}$ in diameter and $100 \mathrm{~mm}$ high probe. The soil and root samples were collected at three distances from the shaft $(10,60$ and $120 \mathrm{~cm})$ and two layers $(0-10$ and 10-20 cm). The roots were separated in two diameter classes: 0 to $2 \mathrm{~mm}$ and 2 to $5 \mathrm{~mm}$. Dry matter of roots were characterized, root radius, length, volume and root area. Rock phosphate did not stimulate root growth and the soluble phosphate was the source that improved growth and development of fine roots in Eucalyptus dunnii, with the highest concentration of these roots being located near the shaft and in the

${ }^{1}$ Universidade do Estado de Santa Catarina, Lages, SC, Brasil.

${ }^{2}$ Universidade Federal de Santa Maria, Santa Maria, RS, Brasil.

${ }^{3}$ Universidade de São Paulo, Piracicaba, SP, Brasil.

*Autor para correspondência <lupatidias@hotmail.com> 
most superficial layer of soil.

KEYWORDS: absorbing roots, eucalyptus, phosphorus fertilization.

\section{INTRODUÇÃO}

O P participa da maioria dos processos vitais do desenvolvimento vegetal (NOVAIS \& SMYTH 1999). Está envolvido em processos essenciais como respiração, fotossíntese e regulação protéica, sendo importante ao estímulo do desenvolvimento do sistema radicular (TAIZ \& ZEIGER 2013). A deficiência de $\mathrm{P}$ compromete a produção de energia, a absorção dos demais nutrientes bem como seu transporte e utilização interna (SINGH et al. 2013), influencia negativamente na morfologia, resultando na redução do potencial produtivo das plantas (PARENTONI et al. 2011).

A exigência de $\mathrm{P}$ pelo eucalipto é alta no ano de implantação e diminui com o aumento da idade. Em razão disso, o nível crítico de $\mathrm{P}$ no solo é alto na fase inicial de desenvolvimento, diminuindo na fase de manutenção da floresta (NOVAIS et al. 1990).

Para solos de baixa fertilidade (como o utilizado no presente estudo) uma sugestão seria a aplicação do $\mathrm{P}$ em um volume maior de solo na forma de fosfato natural, para garantir suprimento às plantas por um período mais longo de tempo devido ao seu efeito residual (BARROS et al. 1990), o que com o passar do tempo pode ser compatível à aplicação dos fosfatos solúveis (GATIBONI et al. 2003, CHIEN et al. 2011), bem como utilizar a aplicação localizada de uma fonte solúvel de $\mathrm{P}$, estimulando assim o desenvolvimento radicular do eucalipto.

Neste contexto, a aplicação de uma fonte natural de $\mathrm{P}$ na forma localizada, em covetas ou no sulco de plantio (forma usual na região sul-brasileira), requer estudos mais aprofundados a fim de conhecer sua dinâmica e atuação na indução do crescimento radicular das plantas de eucaliptos, levando em consideração que os teores de $\mathrm{P}$ natural do solo e os aportados via adubação devem ser suficientes para atender a demanda das plantas em todo o seu ciclo (DIAS et al. 2014).

Além de importante mecanismo de absorção dos recursos edáficos do solo, as raízes são importantes para a captação de nutrientes imóveis como o P. Portanto, presume-se que o crescimento radicular seja regulado pela disponibilidade de P (LYNCH 2007).
As raízes finas de absorção são representadas pelas raízes mais distais e tem sido definidas de acordo com um ponto de corte à base de diâmetro, menores que $2 \mathrm{~mm}$, ao passo que as raízes de diâmetros maiores desempenham funções estruturais de transporte e de armazenamento (McCORMACK et al. 2015).

Algumas das características das raízes com este diâmetro são a maior área de superfície em relação ao volume, a alta densidade de pelos radiculares, pouco ou nenhum desenvolvimento secundário (córtex intacto, pouca suberinização e ausência de cortiça na periderme) (McCORMACK et al. 2015).

A distribuição das raízes no solo é responsável diretamente pelo crescimento das plantas e pode ser influenciada por fatores inerentes ao solo (textura, densidade, fertilidade, entre outros) ou pelas circunstâncias em que a espécie se desenvolve como espaçamento e competição entre árvores (MAURICE et al. 2010, FINÉR et al. 2011). Para EISSENSTAT (1992) e FAHEY \& HUGHES (1994), há maior densidade de raízes finas de absorção nas camadas mais superficiais, devido a maior concentração de $\mathrm{P}$ no solo nestas camadas, o que estimula o crescimento radicular.

Essas raízes aumentam a eficiência na absorção dos nutrientes e, em contrapartida, possuem capacidade de proliferação rápida, atingindo maiores comprimentos. As raízes finas de absorção constituem menos de $1 \%$ da biomassa total da floresta, porém sua produção anual pode contribuir com mais de $50 \%$ na produção líquida total florestal (GONÇALVES \& MELLO 2000).

As raízes crescem preferencialmente nas regiões que contêm concentrações altas e favoráveis de nutrientes, fato comprovado por DREW (1975), que através de um experimento com raízes utilizando membranas especiais e solução nutritiva provou que houve desenvolvimento radicular positivo nos locais com presença de todos os nutrientes e onde houve deficiência de P não ocorreu desenvolvimento radicular satisfatório. Observou ainda que houve maior desenvolvimento radicular onde este nutriente estava disponível.

A aplicação de $\mathrm{P}$ localizado, com orientação no crescimento das raízes, pode gerar pontos negativos no que tange à inadequada exploração de outras regiões no solo, prejudicando a absorção de água e de outros nutrientes pelas plantas, além de atuar comprometendo a integridade da árvore durante a ocorrência de ventos fortes (LANI et al. 1995). 
Diante desses fatos, assim como o observado por SCHUMACHER et al. (2004) em angicovermelho, a adubação fosfatada de maior volume de solo com fosfatos naturais, combinada com fosfato solúvel localizado (REZENDE et al. 1982) parece ser uma técnica recomendável para o plantio de eucalipto em solos pobres em $\mathrm{P}$, propiciando desenvolvimento radicular adequado a melhor absorção de $\mathrm{P}$.

Assim, o objetivo do trabalho foi avaliar a distribuição e as características morfológicas do sistema radicular de Eucalyptus dunnii em diferentes distâncias do fuste e profundidades do solo, em plantas fertilizadas com fosfato solúvel, fosfato natural e a combinação de ambos.

\section{MATERIAL E MÉTODOS}

\section{Área de estudo}

O experimento foi conduzido em um povoamento de Eucalyptus dunnii Maiden no município de Otacílio Costa, SC, implantado em novembro de 2010 , sob as coordenadas geográficas de latitude $27^{\circ} 39^{\prime} 55,775^{\prime \prime} \mathrm{S}$, e longitude $49^{\circ} 45^{\prime} 56,950^{\prime \prime}$ $\mathrm{W}$, cujo clima regional é classificado como $\mathrm{Cfb}$, pela classificação de Köppen.

Mudas clonais de Eucalyptus dunnii (clone KS-25) foram plantadas em espaçamento $2,5 \times 2,5$ $\mathrm{m}$, em área anteriormente utilizada para o cultivo de Pinus taeda, a qual encontrava-se no terceiro ciclo de plantio.

O solo da área do experimento é um Cambissolo Húmico Alumínio léptico (EMBRAPA 2013), formado a partir de materiais resultantes da meteorização de rochas eruptivas da formação Serra Geral (EMBRAPA 2004), e caracterizado segundo metodologia proposta por TEDESCO et al. (1995). Os dados de caracterização química do solo no momento do plantio estão apresentados na Tabela 1 .

\section{Delineamento amostral}

$\mathrm{O}$ delineamento experimental utilizado foi blocos ao acaso, em esquema de parcelas subdivididas, com três repetições. $\mathrm{Na}$ parcela principal, os tratamentos foram: ausência ou presença de fosfato natural na dose de $60 \mathrm{~kg} \mathrm{ha}^{-1} \mathrm{de}_{2} \mathrm{O}_{5}$; e nas subparcelas as doses de fosfato solúvel de 0 ou de 75 $\mathrm{kg} \mathrm{ha}^{-1} \mathrm{P}_{2} \mathrm{O}_{5}$ (doses escolhidas por representar o usual nos reflorestamentos da região).

Tabela 1. Características químicas médias da camada de 0,00-0,20 $\mathrm{m}$ do Cambissolo Húmico da área experimental.

Table 1. Average chemical characteristics of the 0.00-0.20 m layer of the Humic Cambisol of the experimental area.

\begin{tabular}{|c|c|}
\hline Características & Eucalyptus dunnii \\
\hline Argila $\left(\mathrm{g} \mathrm{kg}^{-1}\right)$ & 243 \\
\hline Matéria orgânica $\left(\mathrm{g} \mathrm{kg}^{-1}\right)$ & 54 \\
\hline $\mathrm{pH}$ em $\mathrm{H}_{2} \mathrm{O}(1: 1)$ & 4,1 \\
\hline Acidez potencial $(\mathrm{H}+\mathrm{Al})$ & 35,9 \\
\hline Índice SMP & 4,2 \\
\hline Ca trocável $\left(\mathrm{cmol}_{\mathrm{c}} \mathrm{dm}^{-3}\right)$ & 0,5 \\
\hline $\mathrm{Mg}$ trocável $\left(\mathrm{cmol}_{\mathrm{c}} \mathrm{dm}^{-3}\right)$ & 0,2 \\
\hline $\mathrm{Al}$ trocável $\left(\mathrm{cmol}_{\mathrm{c}} \mathrm{dm}^{-3}\right)$ & 8,5 \\
\hline P disponível Mehlich $1\left(\mathrm{mg} \mathrm{dm}^{-3}\right)$ & 4,9 \\
\hline K disponível Mehlich $1\left(\mathrm{mg} \mathrm{dm}^{-3}\right)$ & 47,0 \\
\hline $\mathrm{K}\left(\mathrm{cmol}_{\mathrm{c}} \mathrm{dm}^{-3}\right)$ & 0,12 \\
\hline $\mathrm{CTC}_{\text {efetiva }}\left(\mathrm{cmol}_{\mathrm{c}} \mathrm{dm}^{-3}\right)$ & 9,2 \\
\hline $\mathrm{CTC}_{\mathrm{pH} 7,0}\left(\mathrm{cmol}_{\mathrm{c}} \mathrm{dm}^{-3}\right)$ & 36,6 \\
\hline Saturação por bases (\%) & 2,0 \\
\hline Saturacão por alumínio (\%) & 91,9 \\
\hline
\end{tabular}


As parcelas principais (com ausência ou presença de fosfato natural) possuíam $312,5 \mathrm{~m}^{2}$ de área útil e continham 50 plantas. Já as subparcelas, que receberam as doses de fosfato solúvel (doses de 0 ou de $75 \mathrm{~kg} \mathrm{ha}^{-1} \mathrm{P}_{2} \mathrm{O}_{5}$ ), mediam $156,25 \mathrm{~m}^{2}$ e continham 25 plantas.

\section{Implantação do experimento}

$\mathrm{O}$ fosfato natural utilizado foi o fosfato de Bayovar, com $34 \%$ de $\mathrm{P}_{2} \mathrm{O}_{5}$ total e $16 \%$ de $\mathrm{P}_{2} \mathrm{O}_{5}$ solúvel em ácido cítrico, determinados segundo método do Ministério da Agricultura Pecuária e Abastecimento (MAPA 2007). A fonte de fosfato solúvel utilizada foi o superfosfato triplo, com $44 \%$ de $\mathrm{P}_{2} \mathrm{O}_{5}$ total e $42 \%$ de $\mathrm{P}_{2} \mathrm{O}_{5}$ solúvel em água.

A adubação fosfatada foi realizada unicamente na época de plantio, e o fosfato solúvel foi aplicado em duas covas distantes cerca de $10 \mathrm{~cm}$ nas laterais de cada planta no sentido da entrelinha de plantio. Os tratamentos com uso de fosfato natural também foram aplicados em duas covas distantes, cerca de 10 $\mathrm{cm}$, nas laterais de cada planta no sentido da linha de plantio.

No plantio das mudas, foi realizada a adubação de base com KCl $\left(30 \mathrm{~kg} \mathrm{ha}^{-1}\right.$ de $\left.\mathrm{K}_{2} \mathrm{O}\right)$ e ureia $(15 \mathrm{~kg}$ $\mathrm{ha}^{-1}$ de N), ambos aplicados na superfície e em área total, juntamente com calcário na dose de $1,0 \mathrm{Mg}$ ha $^{-1}$ (Filler, PRNT de 98,1\%) para suprimento de cálcio $(\mathrm{Ca})$ e magnésio $(\mathrm{Mg})$, tendo como base o manejo florestal utilizado em áreas de florestamento da região. A adubação de cobertura foi realizada em superfície, com aplicação na projeção da copa das plantas. Foram aplicados $60 \mathrm{~kg} \mathrm{ha}^{-1}$ de $\mathrm{K}_{2} \mathrm{O}$ e $30 \mathrm{~kg}$ $\mathrm{ha}^{-1}$ de $\mathrm{N}$, parcelados em duas épocas (aos cinco e aos 10 meses após o plantio).

\section{Coleta}

A coleta de raízes foi realizada 28 meses após o plantio das mudas e como unidade amostral foi escolhida a árvore central das subparcelas (uma árvore por subparcela). Para a coleta do solo com raízes foi utilizada uma sonda cilíndrica de ferro com um tubo de PVC em seu interior, de $30 \mathrm{~cm}$ de comprimento e $40 \mathrm{~mm}$ de diâmetro. Foram coletados seis pontos por árvore, três pontos na linha e três pontos na entrelinha, nas distâncias do fuste de 10, 60 e $120 \mathrm{~cm}$ e na profundidade de 0 a $20 \mathrm{~cm}$.

Os tubos de PVC foram delimitados nos comprimentos de $0-10 \mathrm{~cm}$ e de $10-20 \mathrm{~cm}$, sendo estas as profundidades consideradas. A separação do solo e das raízes foi feita sobre um conjunto de peneiras com uso de pinça, separando raízes finas $(0-2 \mathrm{~mm}$ de diâmetro) e raízes grossas (2 a $5 \mathrm{~mm}$ de diâmetro), após a lavagem do solo. Uma amostra do solo contido no tubo de PVC foi coletada em cada distância e profundidade, para determinação do teor de $\mathrm{P}$ através de extração por resina trocadora de ânions (RTA).

\section{Processamento do solo e raízes}

Foi determinada a massa total úmida (peso úmido) e o peso seco de raízes finas (após secagem em estufa de circulação de ar a $60{ }^{\circ} \mathrm{C}$ ) e de raízes grossas com auxílio de balança analítica. Das raízes finas, foram pesados $0,2 \mathrm{~g}$ e armazenadas em álcool $70 \%$ sob refrigeração para posterior determinação dos parâmetros morfológicos de raízes. Utilizou-se a equação $1, M S=(P U-P S / P U) \times 100$ para determinar a massa seca, sendo MS a massa seca, PU o peso úmido e PS o peso seco.

Os parâmetros morfológicos das raízes finas de absorção $(<2 \mathrm{~mm})$ foram determinados conforme metodologia apresentada por SCHENK \& BARBER (1979). O comprimento (L) de raiz foi determinado pelo método de intersecção descrito por TENNANT (1975). O raio médio da raiz $(\mathrm{R})$ foi calculado pela equação $2, R=(P F / L \pi) 1 / 2$, onde $\mathrm{PF}$ é o peso fresco da raiz. Essa fórmula pressupõe a forma cilíndrica das raízes com densidade de $1,0 \mathrm{~g} \mathrm{~cm}^{3}$. Aárea foi calculada segundo a metodologia de ROSSIELLO et al. (1995) através da equação $3, A=(2 \pi R)$ e o volume pela equação $4, V=(\pi R 2) L$.

\section{Análise dos dados}

Os dados foram submetidos à análise de variância, sendo as médias comparadas pelo teste de Scott-Knott. Foi realizada correlação simples de Pearson entre o teor de P no solo com as demais variáveis. Todas as análises foram conduzidas usando o software Statistical Analysis System ${ }^{\circledR}$ (SAS 2003), considerando o nível mínimo de significância de 5\% .

\section{RESULTADOS}

A análise de variância para massa seca de raízes finas de absorção $(<2 \mathrm{~mm})$ mostrou significância somente para as fontes de $\mathrm{P}$ e para a profundidade de coleta. Não houve respostas significativas para as demais variáveis analisadas e não ocorreu interação entre elas.

O fosfato solúvel foi a fonte de P que ocasionou 
maior produção de massa seca de raízes finas de absorção $(<2 \mathrm{~mm})$ em relação as demais $(0,41 \mathrm{~g}$ $\left.\mathrm{dm}^{-3}\right)$. A testemunha, o fosfato natural e a união de fosfato natural e fosfato solúvel, tiveram produção similares, não apresentando diferenças estatísticas (média de 0,28 $\mathrm{g} \mathrm{dm}^{-3}$ ).

O maior acúmulo de raízes finas de absorção $(<2 \mathrm{~mm})$ ocorreu na profundidade de $0-10 \mathrm{~cm}$, com massa seca cinco vezes maior que na profundidade de 10-20 cm (Tabela 2).

Não houve diferenças estatísticas na massa seca de raízes finas de absorção $(<2 \mathrm{~mm})$ nas diferentes distâncias em relação ao fuste, indicando que nesta idade o sistema radicular das plantas já atingia toda área experimental.

Para a massa seca de raízes grossas ( 2 a $5 \mathrm{~mm}$ de diâmetro) (Tabela 3), houve resultados significativos apenas para as fontes de $\mathrm{P}$ e para profundidade. Os tratamentos com fosfato solúvel e fosfato natural + fosfato solúvel foram os que resultaram em maior produção de massa seca de raízes finas de absorção $(<2$ $\mathrm{mm})\left(0,19\right.$ e $0,14 \mathrm{~g} \mathrm{dm}^{-3}$, respectivamente) comparado com a testemunha e ao fosfato natural, os quais juntos resultaram em uma produção média de $0,08 \mathrm{~g} \mathrm{dm}^{-3}$, indicando que a fonte solúvel e a junção desta com o fosfato natural promovem resultados positivos no crescimento das raízes finas.

Similarmente ao ocorrido para raízes finas de absorção $(<2 \mathrm{~mm})$, a maior massa de raízes de 2 a $5 \mathrm{~mm}$ foi observada na camada de $0-10 \mathrm{~mm}$, não havendo diferença entre as distâncias do fuste.

Para a massa seca total houve resposta às fontes de $\mathrm{P}$ à profundidade e houve interação entre elas. Os tratamentos com fosfato solúvel e fosfato natural + fosfato solúvel apresentaram as maiores produções de massa seca total (média de $0,87 \mathrm{~g} \mathrm{dm}^{-3}$ ) em relação à testemunha $\left(0,63 \mathrm{~g} \mathrm{dm}^{-3}\right)$ e ao fosfato natural $(0,48$ $\left.\mathrm{g} \mathrm{dm}^{-3}\right)$. Para todas as profundidades a produção de massa seca foi maior na camada superficial (dados não apresentados).

A área radicular seguiu a mesma tendência observada para a massa seca de raízes, tendo maior área radicular no tratamento com aplicação de fosfato solúvel. A maior área radicular de raízes finas de absorção $(<2 \mathrm{~mm})$ foi observada na camada de $0-10$ $\mathrm{cm}$, não ocorrendo diferenças entre as distâncias do fuste (Tabela 4).

Já o volume radicular das raízes finas de

Tabela 2. Massa seca de raízes finas ( $<2 \mathrm{~mm})$ de Eucalyptus dunnii submetido às fontes de fósforo em diferentes distâncias do fuste e profundidade do solo.

Table 2. Dry matter of fine roots $(<2 \mathrm{~mm})$ of Eucalyptus dunnii under phosphorus sources at different distances from the stem.

\begin{tabular}{|c|c|c|c|c|c|c|c|}
\hline \multirow[b]{3}{*}{ Fonte $\mathrm{P}$} & \multicolumn{3}{|c|}{ Profundidade $0-10 \mathrm{~cm}$} & \multicolumn{4}{|c|}{ Profundidade $10-20 \mathrm{~cm}$} \\
\hline & \multicolumn{3}{|c|}{ Distância do fuste $(\mathrm{cm})$} & \multicolumn{3}{|c|}{ Distância do fuste $(\mathrm{cm})$} & \multirow[b]{2}{*}{ Média } \\
\hline & 10 & 60 & 120 & 10 & 60 & 120 & \\
\hline \multicolumn{8}{|c|}{$\mathrm{g} \mathrm{dm}^{-3}$} \\
\hline Testemunha & 0,44 & 0,69 & 0,47 & 0,17 & 0,04 & 0,04 & $0,31 \mathrm{~B}$ \\
\hline FS & 0,63 & 0,92 & 0,58 & 0,17 & 0,13 & 0,06 & $0,41 \mathrm{~A}$ \\
\hline FN & 0,35 & 0,40 & 0,39 & 0,12 & 0,06 & 0,03 & $0,22 \mathrm{~B}$ \\
\hline $\mathrm{FN}+\mathrm{FS}$ & 0,53 & 0,46 & 0,53 & 0,17 & 0,07 & 0,06 & $0,30 \mathrm{~B}$ \\
\hline Média & $0,48^{\mathrm{ns}}$ & 0,62 & 0,49 & $0,16^{\mathrm{ns}}$ & 0,07 & 0,05 & \\
\hline \multicolumn{8}{|c|}{ Média da distância } \\
\hline do fuste & $0,32^{\text {ns }}$ & 0,34 & 0,27 & & & & \\
\hline \multicolumn{8}{|l|}{ Média da } \\
\hline profundidade & & $0,53 \mathrm{a}$ & & & $0,09 b$ & & \\
\hline
\end{tabular}

Dentro de cada fator, médias seguidas de letras distintas diferem entre si pelo teste de Scott-Knott a 5\% de significância $(\mathrm{p}<0,05)$. CV $=49,1 \%$.

Inexistência de letras significa ausência de diferenças estatísticas.

ns = não significativo para os níveis testados; FS = fosfato solúvel; FN = fosfato natural. 
Tabela 3. Massa seca de raízes 2-5 mm de Eucalyptus dunnii submetido às fontes de fósforo em diferentes distâncias do fuste e profundidade do solo.

Table 3. Dry matter of roots with 2-5 mm Eucalyptus dunnii under phosphorus sources at different distances from the stem.

\begin{tabular}{|c|c|c|c|c|c|c|c|}
\hline \multirow[b]{3}{*}{ Fonte P } & \multicolumn{3}{|c|}{ Profundidade $0-10 \mathrm{~cm}$} & \multicolumn{3}{|c|}{ Profundidade $10-20 \mathrm{~cm}$} & \multirow[b]{3}{*}{ Média } \\
\hline & \multicolumn{3}{|c|}{ Distância do fuste $(\mathrm{cm})$} & \multicolumn{3}{|c|}{ Distância do fuste $(\mathrm{cm})$} & \\
\hline & 10 & 60 & 120 & 10 & 60 & 120 & \\
\hline \multicolumn{8}{|c|}{$\mathrm{g} \mathrm{dm}^{-3}$} \\
\hline Testemunha & 0,00 & 0,21 & 0,08 & 0,04 & 0,04 & 0,00 & $0,06 \mathrm{~B}$ \\
\hline FS & 0,15 & 0,19 & 0,35 & 0,04 & 0,02 & 0,07 & $0,14 \mathrm{~A}$ \\
\hline $\mathrm{FN}$ & 0,10 & 0,18 & 0,08 & 0,14 & 0,00 & 0,17 & $0,11 \mathrm{~B}$ \\
\hline $\mathrm{FN}+\mathrm{FS}$ & 0,45 & 0,19 & 0,25 & 0,04 & 0,13 & 0,10 & $0,19 \mathrm{~A}$ \\
\hline Média & $0,17^{\mathrm{ns}}$ & 0,17 & 0,19 & 0,06 & 0,05 & 0,08 & \\
\hline \multicolumn{8}{|c|}{ Média da distância } \\
\hline do fuste & $0,12^{\mathrm{ns}}$ & 0,11 & 0,13 & & & & \\
\hline \multicolumn{8}{|l|}{ Média da } \\
\hline profundidade & & $0,18 \mathrm{a}$ & & & $0,06 \mathrm{~b}$ & & \\
\hline
\end{tabular}

Dentro de cada fator, médias seguidas de letras distintas diferem entre si pelo teste de Scott-Knott a 5\% de significância $(\mathrm{p}<0,05)$. CV $=103,7 \%$.

Inexistência de letras significa ausência de diferenças estatísticas.

ns = não significativo para os níveis testados; FS = fosfato solúvel; FN = fosfato natural.

Tabela 4. Área radicular de Eucalyptus dunnii submetido às fontes de fósforo em diferentes distâncias do fuste e profundidade do solo.

Table 4. Root area of Eucalyptus dunnii under phosphorus sources at different distances from the stem.

\begin{tabular}{|c|c|c|c|c|c|c|c|}
\hline \multirow[b]{3}{*}{ Fonte $\mathrm{P}$} & \multicolumn{3}{|c|}{ Profundidade $0-10 \mathrm{~cm}$} & \multicolumn{3}{|c|}{ Profundidade $10-20 \mathrm{~cm}$} & \multirow[b]{3}{*}{ Média } \\
\hline & \multicolumn{3}{|c|}{ Distância do fuste $(\mathrm{cm})$} & \multicolumn{3}{|c|}{ Distância do fuste $(\mathrm{cm})$} & \\
\hline & 10 & 60 & 120 & 10 & 60 & 120 & \\
\hline & \multicolumn{6}{|c|}{$\mathrm{cm}^{2} \mathrm{dm}^{-3}$} & \\
\hline Testemunha & 64,97 & 70,71 & 65,61 & 27,43 & 17,36 & 15,65 & $43,62 \mathrm{~B}$ \\
\hline FS & 71,54 & 86,55 & 67,90 & 29,19 & 28,86 & 21,16 & $50,86 \mathrm{~A}$ \\
\hline FN & 51,57 & 50,93 & 58,91 & 28,17 & 16,66 & 14,18 & $36,73 \mathrm{~B}$ \\
\hline $\mathrm{FN}+\mathrm{FS}$ & 57,87 & 56,60 & 60,67 & 26,18 & 22,48 & 24,74 & $41,42 B$ \\
\hline Média & $61,49^{\text {ns }}$ & 66,20 & 63,27 & $27,74^{\mathrm{ns}}$ & 21,34 & 18,93 & \\
\hline \multicolumn{8}{|c|}{ Média da distância } \\
\hline do fuste & $44,62^{\text {ns }}$ & 43,77 & 41,10 & & & & \\
\hline \multicolumn{8}{|l|}{ Média da } \\
\hline profundidade & & $63,65 \mathrm{a}$ & & & $22,67 b$ & & \\
\hline
\end{tabular}


absorção ( $<2 \mathrm{~mm})$ (Tabela 5) foi maior no tratamento com aplicação de fosfato solúvel e na camada superficial, não havendo diferenças entre as distâncias do fuste.

Os tratamentos com aplicação de fosfato solúvel também apresentaram maior raio radicular de raízes finas do que os demais tratamentos, com maior raio médio na camada de $0-10 \mathrm{~cm}$ de profundidade. Foi observada diferença entre os raios radiculares nas diferentes distâncias do fuste, ocorrendo maior raio nas distâncias mais próximas ao fuste (dados não apresentados).

O comprimento de raízes finas de absorção $(<2$ $\mathrm{mm}$ ) foi significativo apenas para a profundidade, ocorrendo maior comprimento radicular na camada de 0-10 cm. Para as demais variáveis, não houve resposta aos tratamentos e localização de coleta (dados não apresentados).

Quanto aos teores de P no solo em função dos tratamentos (Tabela 6), embora nos tratamentos com adição de fosfato os teores tenham sido numericamente mais elevados do que na testemunha, não houve efeito significativo, em função do elevado coeficiente de variação observado $(C V=292,7 \%)$. Isso pode ter ocorrido porque os fertilizantes foram aplicados em covas, e provavelmente algumas amostras foram retiradas exatamente em cima da cova de adubação. $\mathrm{O}$ único efeito estatisticamente significativo foi a distância do fuste, onde a distância de $10 \mathrm{~cm}$ apresentou, em geral, maior teor de $\mathrm{P}$ que as demais distâncias, o que é esperado, já que os fertilizantes fosfatados foram aplicados próximos às plantas.

Houve correlação negativa entre o teor de $\mathrm{P}$ e a profundidade de amostragem e distância do fuste, confirmando o efeito de localização da adubação fosfatada (Tabela 7). Todas as variáveis relacionadas ao sistema radicular se correlacionaram negativamente com a profundidade de amostragem, confirmando também que o sistema radicular se desenvolveu principalmente na camada de $0-10 \mathrm{~cm}$.

Tabela 5. Volume radicular de Eucalyptus dunnii submetido às fontes de fósforo em diferentes distâncias do fuste e profundidade do solo.

Table 5. Root volume of Eucalyptus dunnii under phosphorus sources at different distances from the stem.

\begin{tabular}{|c|c|c|c|c|}
\hline \multirow[b]{2}{*}{ Fonte de P } & \multicolumn{3}{|c|}{ Distância do fuste (cm) } & \multirow[b]{2}{*}{ Média } \\
\hline & 10 & 60 & 120 & \\
\hline \multicolumn{5}{|c|}{$\begin{array}{l}\text { Profundidade } 0-10 \mathrm{~cm} \\
\qquad \mathrm{~cm}^{3} \mathrm{dm}^{-3}\end{array}$} \\
\hline Testemunha & 1,70 & 2,06 & 1,74 & $1,83 \mathrm{Ab}$ \\
\hline FS & 2,30 & 3,53 & 2,10 & $2,64 \mathrm{Aa}$ \\
\hline FN & 1,23 & 1,21 & 1,35 & $1,26 \mathrm{Ab}$ \\
\hline $\mathrm{FN}+\mathrm{FS}$ & 1,84 & 1,61 & 1,49 & $1,64 \mathrm{Ab}$ \\
\hline Média & $1,77^{\mathrm{ns}}$ & 2,10 & 1,67 & $1,84 \mathrm{~A}$ \\
\hline \multicolumn{5}{|c|}{$\begin{array}{l}\text { Profundidade } 10-20 \mathrm{~cm} \\
\qquad \mathrm{~cm}^{3} \mathrm{dm}^{-3}\end{array}$} \\
\hline Testemunha & 0,39 & 0,17 & 0,14 & $0,24 \mathrm{Ba}$ \\
\hline FS & 0,54 & 0,41 & 0,21 & $0,39 \mathrm{Ba}$ \\
\hline $\mathrm{FN}$ & 0,44 & 0,21 & 0,12 & $0,25 \mathrm{Ba}$ \\
\hline $\mathrm{FN}+\mathrm{FS}$ & 0,57 & 0,25 & 0,24 & $0,35 \mathrm{Ba}$ \\
\hline Média & $0,48^{\mathrm{ns}}$ & 0,26 & 0,18 & $0,31 \mathrm{~B}$ \\
\hline $\begin{array}{l}\text { Média da distância do } \\
\text { fuste }\end{array}$ & $1,13^{\mathrm{ns}}$ & 1,18 & 0,92 & \\
\hline
\end{tabular}

Dentro de cada fator, médias seguidas de letras distintas diferem entre si pelo teste de Scott-Knott a 5\% de significância $(\mathrm{p}<0,05) . \mathrm{CV}=59,6 \%$. Letras maiúsculas comparam as fontes de P em diferentes PROFC e entre as PROFC; Letras minúsculas comparam as fontes de P dentro das mesmas PROFC. Inexistência de letras significa ausência de diferenças estatísticas. ${ }^{\text {ns }}=$ não significativo para os níveis testados; FS = fosfato solúvel; FN = fosfato natural. 
Tabela 6. Teores de fósforo no solo extraído pelo método de resina trocadora de ânions (RTA) em solo cultivado com Eucalyptus dunnii submetido às fontes de fósforo em diferentes distâncias do fuste e profundidade do solo.

Table 6. Phosphorus contents in soil extracted by anion exchange resin method (RTA) in soil cultivated with Eucalyptus dunnii under phosphorus sources.

\begin{tabular}{|c|c|c|c|c|c|c|c|}
\hline \multirow[b]{3}{*}{ Fonte P } & \multicolumn{3}{|c|}{ Profundidade $0-10 \mathrm{~cm}$} & \multicolumn{3}{|c|}{ Profundidade $10-20 \mathrm{~cm}$} & \multirow[b]{3}{*}{ Média } \\
\hline & \multicolumn{3}{|c|}{ Distância do fuste $(\mathrm{cm})$} & \multicolumn{3}{|c|}{ Distância do fuste $(\mathrm{cm})$} & \\
\hline & 10 & 60 & 120 & 10 & 60 & 120 & \\
\hline & \multicolumn{6}{|c|}{$\mathrm{mg} \mathrm{kg}^{-1}$} & \\
\hline Testemunha & 6,23 & 8,04 & 6,50 & 3,94 & 4,01 & 4,12 & $5,47^{\mathrm{ns}}$ \\
\hline FS & 143,02 & 13,91 & 13,25 & 147,11 & 5,60 & 4,65 & 54,59 \\
\hline FN & 5,19 & 6,75 & 5,08 & 2,44 & 2,32 & 2,44 & 4,03 \\
\hline $\mathrm{FN}+\mathrm{FS}$ & 165,38 & 5,92 & 6,43 & 37,76 & 3,45 & 2,76 & 36,95 \\
\hline Média & 79,96 & 8,66 & 7,82 & 47,81 & 3,84 & 3,49 & \\
\hline \multicolumn{8}{|c|}{ Média da distância } \\
\hline do fuste & $63,89 \mathrm{~A}$ & $6,25 \mathrm{~B}$ & $5,65 \mathrm{~B}$ & & & & \\
\hline \multicolumn{8}{|l|}{ Média da } \\
\hline profundidade & & $32,14^{\mathrm{ns}}$ & & & 18,38 & & \\
\hline
\end{tabular}

Dentro de cada fator, médias seguidas de letras distintas diferem entre si pelo teste de Scott-Knott a 5\% de significância $(\mathrm{p}<0,05) . \mathrm{CV}=292,7 \%$.

Inexistência de letras significa ausência de diferenças estatísticas.

ns = não significativo para os níveis testados; FS = fosfato solúvel; $\mathrm{FN}$ = fosfato natural.

Tabela 7. Correlação simples de Pearson entre o teor de fósforo (P solo) e distância do fuste (DF), profundidade de coleta (PROFC), massa seca $<2 \mathrm{~mm}$ (MS $<2$ ), massa seca 2-5 mm (MS2-5), massa seca total (MST), área, volume, raio e comprimento radicular em Eucalyptus dunnii.

Table 7. Pearson correlation between available phosphorus (P soil) and distance from the shaft (DF), the sampling depth (PROFC), dry matter $<2 \mathrm{~mm}$ (MS<2), dry mass 2-5 mm (MS2-5), total dry matter (MST), area, volume, radius and root length in Eucalyptus dunnii.

\begin{tabular}{lccc}
\hline Variáveis & & & \\
\hline & DF & PROFC & P solo \\
\cline { 2 - 4 } DF & - & $0,000^{\mathrm{ns}}$ & $-0,120^{* *}$ \\
PROFC & $0,000^{\mathrm{ns}}$ & - & $-0,010^{\mathrm{ns}}$ \\
MS $<2$ & $-0,006^{\mathrm{ns}}$ & $-0,609^{* *}$ & $0,033^{\mathrm{ns}}$ \\
MS2-5 & $0,000^{\mathrm{ns}}$ & $-0,144^{* *}$ & $-0,001^{\mathrm{ns}}$ \\
MST & $-0,002^{\mathrm{ns}}$ & $-0,576^{* *}$ & $0,017^{\mathrm{ns}}$ \\
Área & $-0,003^{\mathrm{ns}}$ & $-0,718^{* *}$ & $0,032^{\mathrm{ns}}$ \\
Volume & $-0,006^{\mathrm{ns}}$ & $-0,546^{* *}$ & $0,045^{\mathrm{ns}}$ \\
Raio & $-0,044^{\mathrm{ns}}$ & $-0,539^{* *}$ & $0,095^{* *}$ \\
Comprimento & $0,035^{\mathrm{ns}}$ & $-0,138^{* *}$ & $-0,010^{\mathrm{ns}}$ \\
P solo & $-0,013^{\mathrm{ns}}$ & $-0,203^{* *}$ & - \\
\hline
\end{tabular}

${ }^{* *}<0,05 ;{ }^{\text {ns }}=$ não significativo para os níveis testados; $\mathrm{N}=72$; Valores apresentados em $\mathrm{R}^{2}$. 


\section{DISCUSSÃO}

Para todas as variáveis analisadas, com exceção do comprimento radicular, o qual não respondeu às fontes de $\mathrm{P}$, o fosfato solúvel foi a fonte que propiciou características positivas no desenvolvimento do sistema radicular do Eucalyptus dunnii.

A adição conjunta de fosfato natural e fosfato solúvel ocasionou valores intermediários e o fosfato natural não diferiu da testemunha, resultando em características morfológicas indesejáveis, ou seja, sistema radicular menos desenvolvido, caracterizado por menor concentração de raízes, menor volume, área e raio radicular.

Essa melhor resposta do sistema radicular ao uso de fosfato solúvel ocorreu em resposta à zona de enriquecimento de nutriente presente no solo, gerada pela aplicação localizada do fertilizante fosfatado solúvel. Aárea de alta concentração de P foi responsável pelo fornecimento imediato desse nutriente às raízes das plantas, enquanto que nos outros tratamentos (testemunha e fosfato natural), as raízes entraram em contato com áreas de baixa concentração de P. Assim, em resposta ao maior teor de $\mathrm{P}$ disponível, o sistema radicular respondeu em desenvolvimento. A resposta na morfologia do sistema radicular varia de acordo com o elemento que se encontra em deficiência, mas as plantas tendem a concentrar as raízes nas camadas superficiais. Para isso, reduzem o crescimento da raiz principal e alocam seus recursos para o aumento da densidade de raízes laterais e de pelos radiculares (VANCE et al. 2003, LAMBERS et al. 2008).

$\mathrm{O}$ melhor desenvolvimento do sistema radicular em resposta a doses de $\mathrm{P}$ solúvel também foi observado por RIBEIRO et al. (2010), que mesmo em condições de solo adensado afetando negativamente o desenvolvimento das raízes de Eucalyptus grandis, constataram a reversão dessa ocorrência através do fornecimento de doses maiores do nutriente. NOVAIS et al. (1990) também observaram em Eucalyptus grandis intensa proliferação de raízes finas e longas nos locais onde havia maiores concentrações de $\mathrm{P}$, confirmando os resultados obtidos com o Eucalyptus dunnii.

REIS et al. (1985) relatam que o diâmetro e o volume de raízes finas de eucalipto diminuem à medida que aumentam as doses de adubação, sendo que, quanto mais produtivo for o sítio, menos desenvolvido será o sistema de raízes finas de absorção $(<2 \mathrm{~mm})$. De forma análoga, quando o sítio é pouco produtivo, as plantas terão um sistema radicular mais efetivo, capaz de absorver melhor os nutrientes do solo (REIS et al. 1985). Como estratégia para aumentar a aquisição de $\mathrm{P}$ do solo, as plantas deficientes do nutriente tendem a aumentar a relação da massa seca e o comprimento de raiz mais finas, promovendo incremento na área superficial do sistema radicular (MARSCHNER 1995).

Nesse estudo, esse comportamento não se confirmou, pois a maior concentração de raízes ocorreu no tratamento com a fonte solúvel, ou seja, na maior disponibilidade imediata de P. Comportamento semelhante foi relatado por GONÇALVES \& MELLO (2000), em Eucalyptus grandis, que observou que em sítios menos férteis havia menor densidade de raízes. A deficiência de P nas fases de indução e formação das raízes reduz, significativamente, o comprimento das mesmas (SCHWAMBACH et al. 2005), implicando em redução do crescimento das plantas.

De modo geral, foi na profundidade de $0-10 \mathrm{~cm}$ onde se encontraram os maiores teores de $\mathrm{P}$ e maior concentração de raízes. A distância do fuste, quando significativa, mostrou maiores valores dos parâmetros radiculares próximo do fuste. A movimentação do P no solo ocorre principalmente por difusão e a distâncias muito pequenas (NOVAIS \& SMYTH 1999). Por consequência, há a necessidade do $P$ estar localizado próximo ao sistema radicular para ser captado e absorvido. Há relatos que a biomassa de raízes finas diminui exponencialmente da superfície às camadas mais profundas do solo em diferentes biomas florestais (FINÉR et al. 2011), confirmando o encontrado neste estudo, onde houve maior concentração de raízes de absorção nas camadas mais superficiais. Comportamento também observado por BEHLING et al. (2014), quando estudando a formação de raízes finas e médias em Teca, constataram que a densidade, o comprimento e a área radicular específica diminuiram com a profundidade do perfil e os maiores valores concentraram-se na camada de $0-20 \mathrm{~cm}$.

Estudos relatam que $80 \%$ de raízes finas estavam localizadas na profundidade de $0-20 \mathrm{~cm}$ em povoamentos de Eucalyptus grandis e Eucalyptus urophylla aos seis anos de idade (GONÇALVES \& MELLO 2000), semelhante ao observado por CAVALIER (1992) em outras espécies de eucalipto, que relataram que cerca de $85 \%$ das raízes finas se concentraram nos primeiros $20 \mathrm{~cm}$ de solo, ocorrendo redução exponencial na biomassa radicular com o aumento da profundidade. 
No trabalho realizado com Teca, BEHLING et al. (2014) relatam que nos primeiros $20 \mathrm{~cm}$ de solo, concentraram-se $56,2 \%$ da biomassa seca, $57,4 \%$ do comprimento total e $57 \%$ da superfície das raízes finas de Teca $(<2 \mathrm{~mm})$. Trabalhando com Eucalyptus globulus, BAUHUS et al. (2000) constataram maior concentração de raízes finas de absorção até a profundidade de $30 \mathrm{~cm}$. Estas observações reforçam os resultados encontrados neste estudo para o Eucalyptus dunnii, considerando que há correspondência direta entre a maior formação de raízes de absorção nas camadas superficiais do perfil de solo e a maior disponibilidade de água e nutrientes (MAURICE et al. 2010, FINÉR et al. 2011), neste caso especificamente o fósforo.

De forma geral, o fosfato solúvel estimulou a formação de um sistema radicular mais efetivo e com características desejáveis, provocando maior concentração de raízes, maior volume, área e raio radicular de raízes finas quando houve a oferta do $\mathrm{P}$ prontamente disponível no solo, o que não foi observado quando se utilizou adubação com fosfato natural.

Esse comportamento também foi relatado por BEHLING et al. (2014) que trabalhando com desenvolvimento radicular de Teca (Tectona grandis), observaram que o $\mathrm{P}$ e o enxofre são os nutrientes com maior eficiência de utilização para a formação de área radicular. Esta morfologia radicular mais efetiva proporciona maior contato radicular com o $\mathrm{P}$ e resulta em melhoria no aproveitamento dos recursos minerais do sistema, principalmente na absorção de $\mathrm{P}$.

\section{CONCLUSÃO}

O fosfato solúvel proporcionou melhor crescimento de raízes finas de absorção $(<2 \mathrm{~mm})$, ocasionando maior concentração de raízes, maior volume e área no Eucalyptus dunnii, sendo que a maior concentração dessas raízes está localizada nos primeiros centímetros próximos ao fuste e na camada mais superficial do solo, decorrente da presença do $\mathrm{P}$ solúvel. Por outro lado, o uso de fosfato natural não estimulou o desenvolvimento radicular das plantas.

\section{AGRADECIMENTOS}

À CAPES pela concessão da bolsa de doutorado, $\mathrm{CNPq}$ pelo financiamento das bolsas de produtividade e a Klabin S/A pelo incentivo à pesquisa.

\section{REFERÊNCIAS}

BARROS NF et al. 1990. Fertilização e correção do solo para o plantio de eucalipto. In: BARROS NF \& NOVAIS RF (Eds.). Relação solo-eucalipto. Viçosa, MG: Folha de Viçosa. p.127-186.

BAUHUS J et al. 2000. Aboveground and belowground interactions in mixed plantations of Eucalyptus globules and Acacia mearnsii. Canadian Journal of Forest Research 30: 1886-1894.

BEHLING M et al. 2014. Eficiência de utilização de nutrientes para formação de raízes finas e médias em povoamentos de teca. Revista Árvore 38: 837-846.

CAVALIER J. 1992. Fine-root biomass and soil properties in a semideciduous and a lower montane rain forest in Panama. Plant and Soil 142: 187-201.

CHIEN SH et al. 2011. Agronomic and environmental aspects of phosphate fertilizers varying in source and solubility: an update review. Nutrient Cycling in Agroecosystems 89: 229-255.

DIAS LPR et al. 2014. Substituição parcial de fosfato solúvel por natural na implantação de Eucalyptus benthamii e Eucalyptus dunnii no Planalto Sul Catarinense. Revista Brasileira de Ciência do Solo 38: 516-523.

DREW MC. 1975. Comparison of the effects of a localized supply of phosphate, nitrate, ammonium and potassium on the growth of the seminal root system, and the shoot, in barley. New Phytologist 75: 479-490.

EISSENSTAT DM. 1992. Costs and benefits of constructing roots of small diameter. Journal of Plant Nutrition 15: 763782.

EMBRAPA-Empresa Brasileira de Pesquisa Agropecuária. 2004. Solos do Estado de Santa Catarina. Boletim de Pesquisa e Desenvolvimento $\mathrm{n}^{\mathrm{o}}$ 46. Embrapa Solos. Disponível em: http://www.infoteca.cnptia.embrapa.br/ bitstream/doc/664198/1/BOP46.pdf. Acesso em: 10 fev. 2016.

EMBRAPA-Empresa Brasileira de Pesquisa Agropecuária. 2013. Sistema Brasileiro de Classificação de Solos. 3.ed. Embrapa Solos. Disponível em: http://livraria.sct.embrapa. br/liv_resumos/pdf/00053080.pdf. Acesso em: 10 fev. 2016.

FAHEY TJ \& HUGHES JW. 1994. Fine root dynamics in a northern hardwood forest ecosystem, Hubbard Brook Experimental Forest, NH. Journal of Ecology 82: 533-548. FINÉR L et al. 2011. Factors causing variation in fine root biomass in forest ecosystems. Forest Ecology and Management 261: 265-277.

GATIBONI LC et al. 2003. Superphosphate and rock phosphates as phosphorus sources for grass-clover pasture on a limed acid soil of Southern Brazil. Communications in Soil Science and Plant Analysis 34: 2503-2514.

GONÇALVES JLM \& MELLO SLM. 2000. O sistema radicular das árvores. In: GONÇALVES JLM \& BENEDETTI V. (Eds.). Nutrição e fertilização florestal. 
Piracicaba: IPEF. p.219-267.

LAMBERS $\mathrm{H}$ et al. 2008. Plant nutrient-acquisition strategies change with soil age. Trends in Ecology \& Evolution 23: 95-103.

LANI JL et al. 1995. Efeito de doses e localização de fósforo sobre o crescimento de mudas de eucalipto. Revista Ceres 42: 497-506.

LYNCH JP. 2007. Roots of the second green revolution. Australian Journal of Botany 55: 493-512.

MARSCHNER H. 1995. Mineral nutrition of higher plants. 2.ed. San Diego: Academic Press. 889p.

MAURICE J et al. 2010. Fine root isotropy in Eucalyptus grandis plantations. Towards the prediction of root length densities from root counts on trench walls. Plant and Soil 334: 261-275.

MAPA - Ministério da Agricultura e Abastecimento. 2007. Manual de métodos analíticos oficiais para fertilizantes minerais, orgânicos, organominerais e corretivos. Disponível em: http://www.laborsolo.com.br/arquivos/ normativas/INM28.pdf. Acesso em: 18 dez. 2015.

McCORMACK LM et al. 2015. Redefining fine roots improves understanding of below-ground contributions to terrestrial biosphere processes. New Phytologist 207: 505518.

NOVAIS RF \& SMYTH TJ. 1999. Fósforo na planta. In: NOVAIS RF \& SMYTH TJ. (Eds.). Fósforo em solo e planta em condições tropicais. Viçosa: UFV. 399p.

NOVAIS RF et al. 1990. Nutrição Mineral do eucalipto. In: BARROS NF \& NOVAIS RF. (Eds.). Relação soloeucalipto. Viçosa: Editora Folha de Viçosa. 330p.

PARENTONI SN et al. 2011. Melhoramento para eficiência no uso do fósforo. In: FRITSCHE-NETO R \& BOREM A (Eds.). Melhoramento de plantas para condições de estresse abióticos. Visconde do Rio Branco: Suprema. p.101-126. RIBEIRO MAV et al. 2010. Resposta da soja e do eucalipto ao aumento da densidade do solo e a doses de fósforo. Revista Brasileira de Ciência do Solo 34: 1157-1164.

REIS MGF et al. 1985. Acúmulo de biomassa em uma sequência de idade de Eucalyptus grandis plantado no cerrado em duas áreas com diferentes produtividades. Revista Árvore 9: 149-162.

REZENDE GC et al. 1982. Aplicação de fosfatos naturais em plantios de Eucalyptus grandis W. Hill ex maiden. Revista Árvore 6: 74-83.

ROSSIELLO ROP et al. 1995. Comparação dos métodos fotoelétricos e da interseção na determinação de área, comprimento e raio médio radicular. Pesquisa Agropecuária Brasileira 30: 633-638.

SAS Institute Inc ${ }^{\circledR}$. 2003. SAS Ver. 9.1. 3 SAS Institute Inc.: CARY, N.C, USA.

SCHWAMBACH J et al. 2005. Mineral nutrition and adventitious rooting in microcuttings of Eucalyptus globulus. Tree Physiology 25: 487-494.

SCHUMACHER MV et al. 2004. Influência de diferentes doses de fósforo no crescimento de mudas de angico- vermelho (Parapiptadenia rigida (Bentham) Brenan). Revista Árvore 28: 149-155.

SCHENK MK \& BARBER SA. 1979. Phosphate uptake by corn as affected by soil characteristics and root morphology. Soil Science Society of America Journal 43: 880-883.

SINGH SK et al. 2013. Carbon dioxide diffusion across stomata and mesophyll and photo-biochemical processes as affected by growth $\mathrm{CO}_{2}$ and phosphorus nutrition in cotton. Journal of Plant Physiology 170: 801-813.

TAIZ L \& ZEIGER E. 2013. Fisiologia Vegetal. 5.ed. Artmed: Porto Alegre. 918p.

TEDESCO MJ et al. 1995. Análises de solo, planta e outros materiais. 2.ed. Porto Alegre: UFRGS. 174p.

TENNANT D. 1975. A test of a modified line intercept method of estimating root length. Journal of Ecology 63: 995-1001.

VANCE CP et al. 2003. Phosphorus acquisition and use: critical adaptations by plants for securing a nonrenewable resource. New Phytologist 157: 423-447. 